\title{
Leishmania infantum Lipophosphoglycan-Deficient Mutants: A Tool to Study Host Cell-Parasite Interplay
}

OPEN ACCESS

Edited by:

Debora Decote-Ricardo, Universidade Federal Rural do Rio de Janeiro, Brazil

Reviewed by:

Fabienne Tacchini-Cottier,

Université de Lausanne, Switzerland Leonardo Marques da Fonseca, Universidade Federal do Rio de Janeiro, Brazil

*Correspondence:

Albert Descoteaux albert.descoteaux@iaf.inrs.ca Valéria M. Borges

vborges@bahia.fiocruz.br

tThese authors have contributed equally to this work.

¥These authors have contributed equally to this work and as senior author.

Specialty section: This article was submitted to Microbial Immunology, a section of the journal

Frontiers in Microbiology

Received: 13 December 2017 Accepted: 19 March 2018 Published: 05 April 2018

Citation:

Lázaro-Souza M, Matte C, Lima JB, Arango Duque G,

Quintela-Carvalho G, de Carvalho Vivarini Á, Moura-Pontes S,

Figueira $C P$, Jesus-Santos FH,

Gazos Lopes U, Farias LP, Araújo-Santos T, Descoteaux A and

Borges VM (2018) Leishmania infantum Lipophosphoglycan-Deficient Mutants: A Tool to Study Host

Cell-Parasite Interplay.

Front. Microbiol. 9:626. doi: 10.3389/fmicb.2018.00626
Milena Lázaro-Souza ${ }^{1,2 t}$, Christine Matte ${ }^{3 t}$, Jonilson B. Lima ${ }^{4}$, Guillermo Arango Duque ${ }^{3}$, Graziele Quintela-Carvalho ${ }^{1,2,5}$, Áislan de Carvalho Vivarini ${ }^{6}$, Sara Moura-Pontes ${ }^{2}$, Cláudio P. Figueira ${ }^{1}$, Flávio H. Jesus-Santos ${ }^{1,2}$, Ulisses Gazos Lopes ${ }^{6}$, Leonardo P. Farias ${ }^{1}$, Théo Araújo-Santos ${ }^{4}$, Albert Descoteaux ${ }^{3 * \pm}$ and Valéria M. Borges ${ }^{1,2 * \neq}$

1 Laboratory of Inflammation and Biomarkers, Gonçalo Moniz Institut, Oswaldo Cruz Foundation, Salvador, Brazil,

${ }^{2}$ Department of Legal Medicine, Federal University of Bahia, Salvador, Brazil, ${ }^{3}$ Institut National de la Recherche Scientifique -Institut Armand-Frappier, Laval, QC, Canada, ${ }^{4}$ Center of Biological Sciences and Health, Federal University of Western of Bahia, Barreiras, Brazil, ${ }^{5}$ Instituto Federal de Educação, Ciência e Tecnologia Baiano (IFBaiano), Alagoinhas, Brazil, ${ }^{6}$ Laboratory of Molecular Parasitology, Carlos Chagas Filho Biophysics Institute, Center of Health Science, Universidade Federal do Rio de Janeiro, Rio de Janeiro, Brazil

Lipophosphoglycan (LPG) is the major surface glycoconjugate of metacyclic Leishmania promastigotes and is associated with virulence in various species of this parasite. Here, we generated a LPG-deficient mutant of Leishmania infantum, the foremost etiologic agent of visceral leishmaniasis in Brazil. The L. infantum LPG-deficient mutant $(\Delta / \mathrm{pg} 1)$ was obtained by homologous recombination and complemented via episomal expression of LPG1 ( $\triangle / p g 1+\angle P G 1)$. Deletion of $L P G 1$ had no observable effect on parasite morphology or on the presence of subcellular organelles, such as lipid droplets. While both wild-type and add-back parasites reached late phase in axenic cultures, the growth of $\triangle / p g 1$ parasites was delayed. Additionally, the deletion of LPG1 impaired the outcome of infection in murine bone marrow-derived macrophages. Although no significant differences were observed in parasite load after $4 \mathrm{~h}$ of infection, survival of $\Delta / p g 1$ parasites was significantly reduced at $72 \mathrm{~h}$ post-infection. Interestingly, L. infantum LPG-deficient mutants induced a strong NF-кB-dependent activation of the inducible nitric oxide synthase (iNOS) promoter compared to wild type and $\Delta / p g 1+\angle P G 1$ parasites. In conclusion, the $L$. infantum $\Delta / p g 1$ mutant constitutes a powerful tool to investigate the role(s) played by LPG in host cell-parasite interactions.

Keywords: Lipophosphoglycan, Leishmania infantum, gene targeting, lipid droplets, macrophage

\section{INTRODUCTION}

Lipophosphoglycan (LPG) is one of the most abundant components of Leishmania membranes (Turco and Descoteaux, 1992). In the course of parasite interaction with invertebrate hosts, LPG binds to the midgut epithelium of specific species of the sandfly vectors (Sacks et al., 2000), and protects parasites against the digestive enzymes present in the peritrophic matrix following blood feeding (Sacks and Kamhawi, 2001). In vertebrate hosts, LPG contributes to virulence by shielding Leishmania against the complement system (Spath et al., 2003) and by inhibiting phagolysosomal 
biogenesis (Desjardins and Descoteaux, 1997; Vinet et al., 2009; Moradin and Descoteaux, 2012). Purified LPG has been considered as a pathogen-associated molecular pattern molecule (PAMP) that triggers Toll-like receptors (TLR) and is also known to interfere with pro-inflammatory and signaling pathways in host cells (Descoteaux et al., 1991; Descoteaux and Turco, 1993; Becker et al., 2003; de Veer et al., 2003; Kavoosi et al., 2009; Rojas-Bernabé et al., 2014; Tavares et al., 2014; Lima et al., 2017).

This complex glycolipid is organized in four domains: a conserved 1-O-alkyl-2-lyso-phosphatidyl(myo)inositol membrane anchor, a conserved diphosphoheptasaccharide core structure, a polymer of repeating phosphodisaccharide units (phosphoglycan or PG) carrying species-specific side chains and variable, often mannose-rich cap structures (Turco and Descoteaux, 1992; McConville and Ferguson, 1993). Although the biosynthesis of LPG has attracted considerable interest, to date only few enzymes and transporters involved in this process have been identified either biochemically, genetically, or both (Ryan et al., 1993; Descoteaux et al., 1995, 1998, 2002).

One of the key enzymes in the biosynthesis of LPG is LPG1, a putative galactofuranosyl transferase specifically involved in the synthesis of the LPG glycan core (Ryan et al., 1993). Consequently, parasites lacking the LPG1 gene $(\Delta l p g 1)$ express a truncated LPG without the PG domain; they nonetheless assemble and secrete other PG-containing molecules (Dermine et al., 2000; Späth et al., 2000). Both L. major and $L$. donovani require LPG1 for the establishment of infection within macrophages, as evidenced by the elimination of LPG1null mutants following phagocytosis; yet, restoration of LPG expression by genetic complementation restored the capacity to replicate within macrophages (Späth et al., 2000; Lodge et al., 2006). Interestingly, phosphoglycan synthesis does not seem to be an absolute requirement for virulence in all Leishmania species, since L. mexicana phosphoglycan-deficient parasites were found to be similarly virulent to their wild-type (WT) counterparts (Ilg et al., 1999, 2001; Ilg, 2000). This difference in LPG requirement for the establishment of infection within macrophages may be related to the fact that $L$. mexicana resides in large fusogenic communal vacuoles, as opposed to the non-fusogenic, tight individual vacuoles in which $L$. major and $L$. donovani replicate. The role played by LPG1 in L. infantum infectivity in mammals remains to be established.

This report describes the disruption of LPG1 in L. infantum, the main etiological agent of visceral leishmaniasis in Brazil. While deletion of LPG1 did not alter parasite morphology in vitro or the presence of subcellular organelles, e.g., lipid droplets (LD), $\Delta l_{p g} 1$ parasites experienced distinct infection outcomes in comparison to WT parasites. Hence, the L. infantum LPG1-null strain described in the present study constitutes a powerful tool to investigate the role of LPG in host-parasite interactions.

\section{METHODS}

\section{Ethics Statement}

This study was carried out in accordance with the recommendations of Institutional Review Board for Animal Experimentation (CEUA), Gonçalo Moniz Institute, Fundação
Oswaldo Cruz. The protocol was approved by the Institutional Review Board for Animal Experimentation (CEUA), Gonçalo Moniz Institute, Fundação Oswaldo Cruz (Protocol No. 021/2015).

\section{Animals}

Inbred male C57BL/6 mice, aged 6-8 weeks, were obtained from the animal care facility of the Gonçalo Moniz Institute, Fundação Oswaldo Cruz (IGM-FIOCRUZ, Bahia, Brazil).

\section{Targeted Deletion of the LPG1 Gene and Complementation}

The constructs for LPG1 (beta galactofuranosyl transferase) gene targeting were designed based on the L. infantum LPG1 gene sequence (GenBank accession No. GU233511). Homozygous $L P G 1$-null mutants $(\triangle \operatorname{lpg} 1)$ were obtained using two targeting constructs (Figures 1A,B). For the NEO targeting construct, the entire LPG1 gene was amplified by PCR from $L$. infantum BH46 (MCAN/BR/89/BH46) DNA using Taq DNA polymerase (New England Biolabs) and oligodeoxynucleotides AD-358 (forward) (5'-gtacaagcttccatATGGCGCCGCCTCGCTG-3') and AD-359 (reverse) (5'-gctactcgagTTAGCTGGGGTCAACAG-3'). This fragment was digested with HindIII and XhoI, and then ligated with the HindIII-XhoI-digested pBluescript II SK ${ }^{-}$vector, yielding pBS-LPG1. The NEO resistance cassette from pLeishNeo (unpublished) was extracted with NotI and EcoRV, blunted and inserted in the MscI site of pBS-LPG1, within the LPG1 gene, yielding pBS-LPG1::NEO. For the HYG targeting construct, nucleotides $1-437$ of the LPG1 gene were amplified by RT-PCR from L. infantum BH46 mRNA using oligodeoxynucleotides AD53 (forward) (5'-cgggatccatATGGCGCCGCCTCGCTG-3') and AD-357 (reverse) (5'-ggaattcTCGGGGTGGTGAATG-3'). This fragment was digested with $B a m \mathrm{HI}$ and EcoRI, and then ligated with the BamHI-EcoRI-digested pBluescript II SK ${ }^{-}$vector. A 467-bp fragment containing nucleotides 781-1,247 of the LPG1 ORF was amplified by PCR from L. infantum BH46 genomic DNA using oligodeoxynucleotides AD-355 (forward) (5' gcaagcttGGCATCTATTACACAGACCACAAGG-3') and AD356 (reverse) (5'-caggtcgacTGGCAGCGAATGTTTTCACC-3'). This fragment was digested with HindIII and SalI, then ligated with the same vector, downstream of the first LPG1 sequence, at the HindIII and SalI restriction sites. The HYG resistance cassette from pX63-HYG was excised with SalI and BamHI, blunted and inserted between the two LPG1 sequences, at the EcoRV restriction site, yielding pBS-LPG1::HYG. For genetic complementation of the $\Delta l p g 1$ mutant, the entire LPG1 ORF was amplified by PCR from L. infantum BH46 genomic DNA using Native $P f u$ polymerase (Stratagene, La Jolla, CA, USA) and oligodeoxynucleotides AD-358 (forward) ( $5^{\prime}$-gtacaagcttccatATGGCGCCGCCTCGCTG-3') and AD-359 (reverse) (5'-gctactcgagTTAGCTGGGGTCAACAG-3'). This fragment was digested with HindIII and XhoI, and then ligated with the HindIII-XhoI-digested pBluescript II $\mathrm{SK}^{-}$vector, yielding pBSII-LPG1. The absence of mutations in the amplified LPG1 ORF was verified by Sanger sequencing (Génome Québec; GenBank accession No. GU233511). The LPG1 gene was then excised from pBSII-LPG1 with EcoRV and XhoI, blunted and 


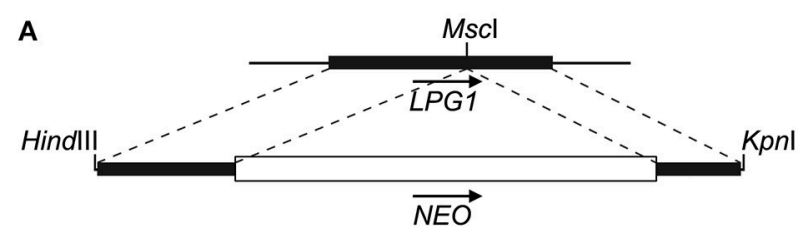

B

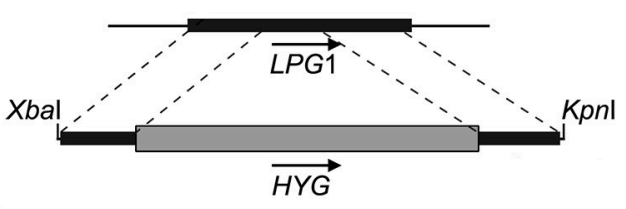

$0.5 \mathrm{~kb}$

D
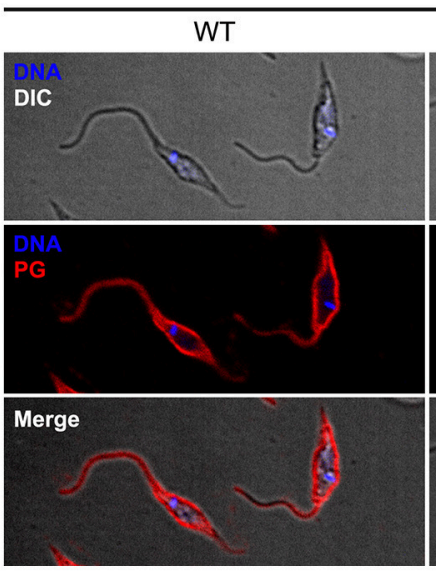

L. infantum BA262

(1)
C

$3.8 \mathrm{~kb}$

$2.9 \mathrm{~kb}$

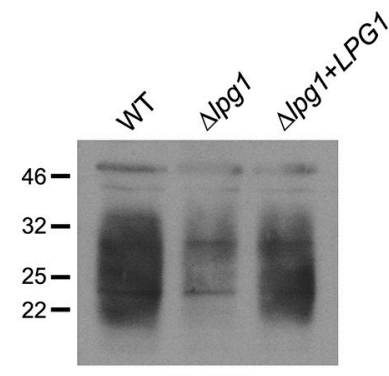

IB: PG $\Delta / p g 1 \quad \Delta / p g 1+L P G 1$

FIGURE 1 | Constructs for the targeted deletion and complementation of the LPG1 gene in Leishmania infantum. (A,B) LPG1::NEO and LPG1::HYG targeting constructs for the disruption of LPG1. For the LPG1::NEO construct, the NEO resistance cassette (white box) was inserted in the Mscl site of the LPG1 ORF (black rectangle). In the LPG1::HYG construct, portions of the LPG1 ORF (black rectangles) corresponding to positions +1 to +437 and to positions +781 to +1247 downstream of the ATG translation initiation codon flank the HYG resistance cassette (shaded rectangle). Dashed lines delimit regions of recombination between the LPG1 gene and the targeting constructs. Arrows indicate gene orientation. (C) Western blot analysis of LPG expression in WT, $\Delta / p g 1$, and $\Delta / p g 1+L P G 1$ promastigotes. Parasite lysates were probed with the anti-phosphoglycan (PG) antibody CA7AE, as described in Materials and Methods. (D) Confocal immunofluorescence analysis of WT, $\Delta / p g 1$ and $\Delta / p g 1+L P G 1$ parasites. Late log-phase promastigotes were adhered on Poly-L-Lysine-coated glass coverslips, fixed and incubated with DAPI to stain DNA (blue), and with the CA7AE antibody to visualize LPG and other Gal( $(\beta 1,4)$ Man $(\alpha 1-P O 4)$ repeating unit-containing PGs (red), as described in Materials and Methods. Fluorescence staining images merged with differential interference contrast (DIC) are shown in the lower panels. Scale bar, $5 \mu \mathrm{m}$.

ligated with the EcoRV-digested pLeishZeo vector (unpublished), yielding pLeishZeo-LPG1.

\section{Transfection and Selection of $L$. infantum $\Delta$ lpg1 Promastigotes}

Log-phase WT L. infantum BA262 (MCAN/BR/89/BA262) promastigotes were first electroporated with the purified $L P G 1:: H Y G$ targeting construct (excised as a 2.9-kb XbaI-KpnI fragment from pBS-LPG1::HYG) using $0.2 \mathrm{~cm}$ electroporation cuvettes, at $0.45 \mathrm{kV}$ and a high capacitance of $500 \mu \mathrm{F}$ as previously described (Turco et al., 1994). Following electroporation, promastigotes were incubated for $24 \mathrm{~h}$ in drug-free, complete M199 medium and subsequently grown in the presence of $50 \mu \mathrm{g} / \mathrm{mL}$ Hygromycin B (Roche Diagnostics). To generate LPG1-null mutants, log-phase $\operatorname{lpg} 1^{+/ H Y G}$ heterozygous L. infantum BA262 promastigotes were electroporated with purified $L P G 1:: N E O$ targeting construct (excised as a $3.8-\mathrm{kb}$ HindIII-KpnI fragment from pBS-LPG1::NEO) and grown after $24 \mathrm{~h}$ in the presence of both $50 \mu \mathrm{g} / \mathrm{mL}$ Hygromycin B and $70 \mu \mathrm{g} / \mathrm{mL}$ G418 (Life Technologies). Absence of LPG in the resulting double drug-resistant $\Delta l p g 1$ promastigotes was verified by Western blot analysis and confocal immunofluorescence. To restore LPG1 expression, log-phase L. infantum BA262 $\Delta l p g 1$ cells were electroporated with pLeishZeo-LPG1. Complemented mutants $(\triangle l p g 1+L P G 1)$ were selected with $80 \mu \mathrm{g} / \mathrm{mL}$ Zeocin (in addition to G418 and Hygromycin B at concentrations specified above) and verified by Western blotting and confocal immunofluorescence.

\section{Parasite Cultures}

L. infantum promastigotes were cultured in HOMEM medium supplemented with 10\% inactivated Fetal Bovine Serum (FBS), 
$100 \mathrm{U} / \mathrm{mL}$ penicillin, $100 \mu \mathrm{g} / \mathrm{mL}$ streptomycin and $2 \mathrm{mM} \mathrm{L}$ glutamine in $25 \mathrm{~cm}^{2}$ flasks at $24^{\circ} \mathrm{C}$ until late log-phase. For L. infantum BA262 $\Delta l p g 1$, Hygromycin $(50 \mu \mathrm{g} / \mathrm{mL})$ and $\mathrm{G} 418(70 \mu \mathrm{g} / \mathrm{mL})$ were added to the medium. For $L P G 1$-complemented parasites $(\Delta l p g 1+L P G 1)$, Hygromycin $(50 \mu \mathrm{g} / \mathrm{mL}), \mathrm{G} 418(70 \mu \mathrm{g} / \mathrm{mL})$, and Zeocin $(100 \mu \mathrm{g} / \mathrm{mL})$ were added to the medium.

\section{Western Blotting}

Late log-phase promastigotes were washed with ice-cold PBS containing $1 \mathrm{mM} \mathrm{Na} \mathrm{VO}_{4}$, then lysed in $50 \mathrm{mM}$ Tris- $\mathrm{HCl}$ $\mathrm{pH} 8,150 \mathrm{mM} \mathrm{NaCl}$ and $1 \%$ Nonidet P-40, containing complete protease inhibitors (Roche Applied Science) and phosphatase inhibitors $\left(1 \mathrm{mM} \mathrm{Na} \mathrm{VO}_{4}, 50 \mathrm{mM} \mathrm{NaF}, 1.5 \mathrm{mM}\right.$ EGTA and $10 \mathrm{mM} \mathrm{Na} \mathrm{P}_{2} \mathrm{O}_{7}$ ). Samples were sonicated briefly, and insoluble material was removed by centrifugation for $10 \mathrm{~min}$ at $4^{\circ} \mathrm{C}$. Protein concentrations were determined using the Pierce BCA protein assay kit (Pierce). Proteins were separated by SDS-PAGE and then transferred to HybondLFP PVDF membranes (GE Healthcare Life Sciences) using a Trans-Blot SD Semi-Dry Transfer Cell apparatus (BioRad). Membranes were blocked with 5\% BSA and incubated with the mouse monoclonal antibody CA7AE (MediMabs). For immunodetection, goat anti-mouse IgM Heavy Chain Secondary antibody conjugated with horseradish peroxidase (HRP), and enhanced chemiluminescence (ECL) detection reagents from GE Healthcare Life Sciences were used.

\section{Confocal Immunofluorescence Microscopy}

Late log-phase promastigotes were adhered on Poly-L-Lysinecoated glass coverslips (BD Biosciences, San Jose, CA) by centrifugation, fixed with $4 \%$ paraformaldehyde (Canemco and Marivac) for $20 \mathrm{~min}$ and simultaneously blocked and permeabilized with a solution of $0.1 \%$ Triton X-100, $1 \%$ BSA, $6 \%$ non-fat dry milk, $20 \%$ goat serum and 50\% FBS for $20 \mathrm{~min}$. The distribution of LPG and other PGs containing the $\operatorname{Gal}(\beta 1,4) \operatorname{Man}\left(\alpha 1-\mathrm{PO}_{4}\right)$ repeating unit epitope was visualized using the mouse monoclonal antibody CA7AE (MediMabs, 1:2,000) after $2 \mathrm{~h}$ incubation followed by Alexa Fluor 568 goat anti-mouse IgM (Molecular Probes) at 1:500 for $30 \mathrm{~min}$ incubation. Parasite nuclei were stained with DAPI (Molecular Probes) at 1:17,000. All steps were performed at room temperature. Coverslips were then mounted in Fluoromount$\mathrm{G}$ (Interscience) and sealed with nail polish. Promastigotes were observed with a Plan APOCHROMAT 63x oil-immersion DIC 1.4 NA objective on a Zeiss LSM780 confocal microscope equipped with a $30 \mathrm{~mW} 405 \mathrm{~nm}$ diode laser, $25 \mathrm{~mW} \mathrm{458/488/514}$ argon multiline laser, $20 \mathrm{~mW}$ DPSS $561 \mathrm{~nm}$ laser and $5 \mathrm{~mW}$ $\mathrm{HeNe} 633 \mathrm{~nm}$ laser, coupled to a Zeiss Axio Observer Z1. Images were acquired in plane scanning mode, and were minimally and equally processed using Carl Zeiss ZEN 2011 software.

\section{Electron Microscopy}

Late log-phase promastigotes were fixed with $2 \%$ paraformaldehyde plus $2.5 \%$ glutaraldehyde in $0.1 \mathrm{M}$ phosphate buffer ( $\mathrm{pH} 7.4$ ) overnight at $4^{\circ} \mathrm{C}$. Next, parasites were processed for Transmission Electron Microscopy (TEM) by post-fixing in $1 \%$ osmium tetroxide $\left(\mathrm{OsO}_{4}\right)$ plus $0.8 \%$ potassium ferricyanide in $0.1 \mathrm{M}$ cacodylate buffer, $\mathrm{pH} 7.2$, then dehydrated in acetone at increasing concentrations of 50, 70, 90, and 100\% followed by processing for resin embedding (PolyBed 812, Polysciences). Sections were mounted on uncoated 200-mesh copper grids and viewed under a TEM microscope (JEOL JEM-1230). Alternatively, parasites were processed for Scanning Electron Microscopy (SEM) by first fixing as described above, then adhered on Poly-L-Lysine-coated glass coverslips and postfixed as described above. Samples were then submitted to critical point-drying under $\mathrm{CO}_{2}$, coated with a $20 \mathrm{~nm}$-layer of gold particles and examined under SEM (JSM-6390LV, JEOL).

\section{Parasite Growth Curves}

Early log-phase promastigotes $\left(1 \times 10^{5} / \mathrm{ml}\right)$ were cultured and the number of viable promastigotes was determined by daily direct counting performed in a Neubauer chamber.

\section{Lipid Droplets Staining and Quantification}

Late log-phase promastigotes were fixed with 3.7\% formaldehyde and stained with osmium tetroxide. Cell morphology was observed, and LD were counted by light microscopy using a 100X objective lens in 50 consecutively scanned parasites (AraújoSantos et al., 2014).

\section{Bone Marrow-Derived Macrophages (BMDM) Macrophage and Infection}

Bone marrow-derived macrophages (BMDM) were obtained from C57BL/6 mice as previously described. Briefly, cells were collected from femurs and differentiated in RPMI 1640, 20\% inactivated FBS, 30\% L929 cell-conditioned media (LCCM), $2 \mathrm{mM}$ L-glutamine, $100 \mathrm{U} / \mathrm{mL}$ Penicillin, and $100 \mu \mathrm{g} / \mathrm{mL}$ Streptomycin at $36^{\circ} \mathrm{C}$ under $5 \% \mathrm{CO}_{2}$. BMDMs were collected after 7 days and seeded on tissue culture plates in RPMI 1640 media, $10 \%$ inactivated FBS, 5\% LCCM and $2 \mathrm{mM}$ L-glutamine (Araújo-Santos et al., 2014).

Cells $\left(2 \times 10^{5}\right)$ adhered on coverslips were infected with either WT, $\Delta \operatorname{lpg} 1$, or $\Delta \operatorname{lpg} 1+L P G 1$ parasites at a $10: 1$ multiplicity of infection (MOI). After 4 or $72 \mathrm{~h}$ of infection, coverslips were fixed and stained with DiffQuik (Wright-Giemsa). Intracellular parasites were counted under light microscopy to determine the infection index under each experimental condition (AraújoSantos et al., 2014).

\section{RAW 264.7 Cell Line, Culture, and Infection}

The mouse macrophage leukemia cell line RAW 264.7 (TIB71; American Type Culture Collection (ATCC), Manassas, VA, USA) was maintained in DMEM medium with high glucose (Vitrocell Embriolife, Campinas, SP, Brazil) supplemented with $10 \%$ heat-inactivated fetal bovine serum (Sigma-Aldrich, St. Louis, MO, USA), $100 \mathrm{U} / \mathrm{ml}$ penicillin and $100 \mu \mathrm{g} / \mathrm{ml}$ streptomycin in an incubator at $37^{\circ} \mathrm{C}$ under $5 \% \quad \mathrm{CO}_{2}$. RAW 264.7 cells were infected with either WT, $\Delta$ lpg1 or $\Delta l p g 1+L P G 1$ parasites at a 10:1 multiplicity of infection (MOI). After 4 or $8 \mathrm{~h}$ of infection, cells were processed 
for quantitative RT-PCR. For the luciferase reporter assay, cultures were washed $2 \mathrm{~h}$ post-infection and analyzed $24 \mathrm{~h}$ later.

\section{RNA Extraction and RT-qPCR}

For real time quantitative polymerase chain reaction analysis, total RNA of control and infected RAW 264.7 cells $(1 \times$ $10^{6}$ cells) was extracted using an Invitrap ${ }^{\circledR}$ Spin Cell RNA mini kit (STRACTEC Molecular GmbH, Berlin, Germany). RNA extracts $(2 \mu \mathrm{g})$ were reverse transcribed into first-strand cDNA with ImProm-II (Promega) and oligo(dT) primers in accordance with manufacturer instructions. The following primer DNA sequences were used to determine iNOS mRNA levels: Forward $5^{\prime}$-CAGCTGGGCTGTACAAACCTT- $3^{\prime}$ and Reverse: $\quad 5^{\prime}$-CATTGGAAGTGAAGCGTTTCG- $3^{\prime}$, while GAPDH mRNA levels were quantified using: Forward $5^{\prime}$-TGCACCACCAACTGCTTAGC-3' and Reverse $5^{\prime}$ GGCATGGACTGTGGTCATGAG-3'. Amplicon specificity was carefully verified by the presence of a single melting temperature peak in dissociation curves calculated following RT-qPCR, which was performed via the Applied Biosystems StepOne ${ }^{\mathrm{TM}}$ detection system (Applied Biosystems) using GoTaq ${ }^{\circledR}$ qPCR Master Mix (Promega Corp., Madison, WI, USA). All RT-qPCR analyses were performed in triplicate. RT-qPCR data was normalized using GAPDH primers as an endogenous control. All gene expression ratios were calculated by the $\Delta \Delta \mathrm{Ct}$ method using StepOne software version 2.0 (Applied Biosystems).

\section{Transient Transfections and Luciferase Assays}

To investigate NF-кB transcriptional activity, RAW 264.7 were plated in 48 -well polystyrene plates $\left(1 \times 10^{5}\right.$ cells per well $)$ and transfected with $1 \mu \mathrm{g}$ of the p6kB-Luc luciferase reporter construct (kindly provided by Dr. Patrick Baeuerle, Munich University) in the presence of LIPOFECTAMINE 2000 reagent (Invitrogen, Carlsbad, CA, USA). pTK-3XNS luciferase reporter construct was used to measure iNOS promoter activity, provided by Dr. David Geller (University of Pittsburgh, Pennsylvania, EUA). Luciferase activity was normalized using 40 ng of pRLCMV plasmid (Promega Corp., Madison, WI, USA). Transfected cells were infected with either WT, $\Delta l p g 1$ or $\Delta l p g 1+L P G 1$ parasites at a 10:1 MOI. After $24 \mathrm{~h}$ of infection, cells were washed with PBS, lysed according to the Dual Luciferase System protocol (Promega Corp.), and analyzed in a GloMax ${ }^{\circledR}$. Multi detection system (Promega Corp.). Positive controls consisting of cells stimulated with $1 \mu \mathrm{g} / \mathrm{mL}$ of LPS (SigmaAldrich) were used to induce the activation of iNOS gene expression.

\section{Statistical Analysis}

BMDM and RAW 264.7 cell infection assays were performed in triplicate, and each experiment was repeated at least three times. Data are presented as the mean and SE (standard error) of representative experiments, and GraphPad Prism 5.0 software (GraphPad Software) was used for data analysis.
Means from different groups were compared by Oneway ANOVA and comparisons between two groups were performed using the Student Newman-Keuls post-test. Differences were considered statistically significant when $p$ $\leq 0.05$.

\section{RESULTS}

\section{Generation of a $L$. infantum Lpg1-Null $\Delta / p g 1$ Mutant}

To generate a $L$. infantum LPG-defective $(\Delta l p g 1)$ mutant, WT $L$. infantum BA262 promastigotes were transfected with the LPG1 targeting constructs (Figures 1A,B). The resulting HYGand NEO-resistant $\triangle l p g 1$ parasites were transfected with a $L P G 1$ expression vector to generate add-back LPG-expressing parasites $(\triangle l p g 1+L P G 1)$. Loss of LPG expression in the $\Delta l p g 1$, was determined by comparing LPG levels in WT, $\Delta l p g 1$, and $\triangle l p g 1+L P G 1 L$. infantum promastigotes by Western blot and by confocal immunofluorescence microscopy (Figures 1C,D). Together, these data indicate that the LPG1 gene was successfully deleted in the $\triangle l p g 1$ mutants, resulting in the generation of a LPG-defective L. infantum mutant.

\section{LPG1-Null Mutants Retain L. infantum Viability and Morphology}

To determine the effect of deleting LPG1 on parasite growth and morphology, axenic cultures of the three isolates were monitored and counted daily for 10 days until reaching late $\log$ phase. A delayed replication capability of the $\Delta l p g 1$ mutant parasites was noted in comparison to the WT and $\Delta l p g 1+$ LPG1 parasites (Figure 2A). Wild-type $L$. infantum presented regular growth for 7 days until reaching stationary phase, with a cell density of approximately $3-4 \times 10^{7}$ parasites $/ \mathrm{ml}$, while the $\Delta l p g 1$ mutant reached the same phase approximately 3 days later, with a cell density of $1-2 \times 10^{7}$ parasites/ml (Figure 2A). The $\triangle l p g 1+L P G 1$ mutants presented an intermediate growth profile, reaching stationary phase shortly after the WT parasites. Area Under the Curve (AUC) analysis of the growth curve revealed a significant difference only when comparing WT and $\Delta l p g 1$ parasites $(p<0.05)$, yet no differences were observed between WT and $\triangle l p g 1+L P G 1$ mutants (Figure 2B). Upon reaching stationary phase, parasites were examined by electron microscopy to assess the presence of morphological alterations. Under both SEM and TEM, no alterations in morphology (Figure 2C) or in ultrastructural characteristics (Figure 3A) were detected among WT, $\Delta l p g 1$, and $\Delta l p g 1+$ LPG1 promastigotes. In addition, the absence of LPG1 had no impact on the number of lipid bodies present within the parasites (Figure 3A). Hence, whereas the LPG1 gene had a limited impact on L. infantum promastigotes proliferation, it did not significantly alter morphological features of these parasites (Figure 3).

\section{Lpg1-Null Mutants Exhibit Limited Survival in Macrophages}

To evaluate differences in parasite survival among WT and transgenic parasites in vitro, BMDMs were infected for 4 or 

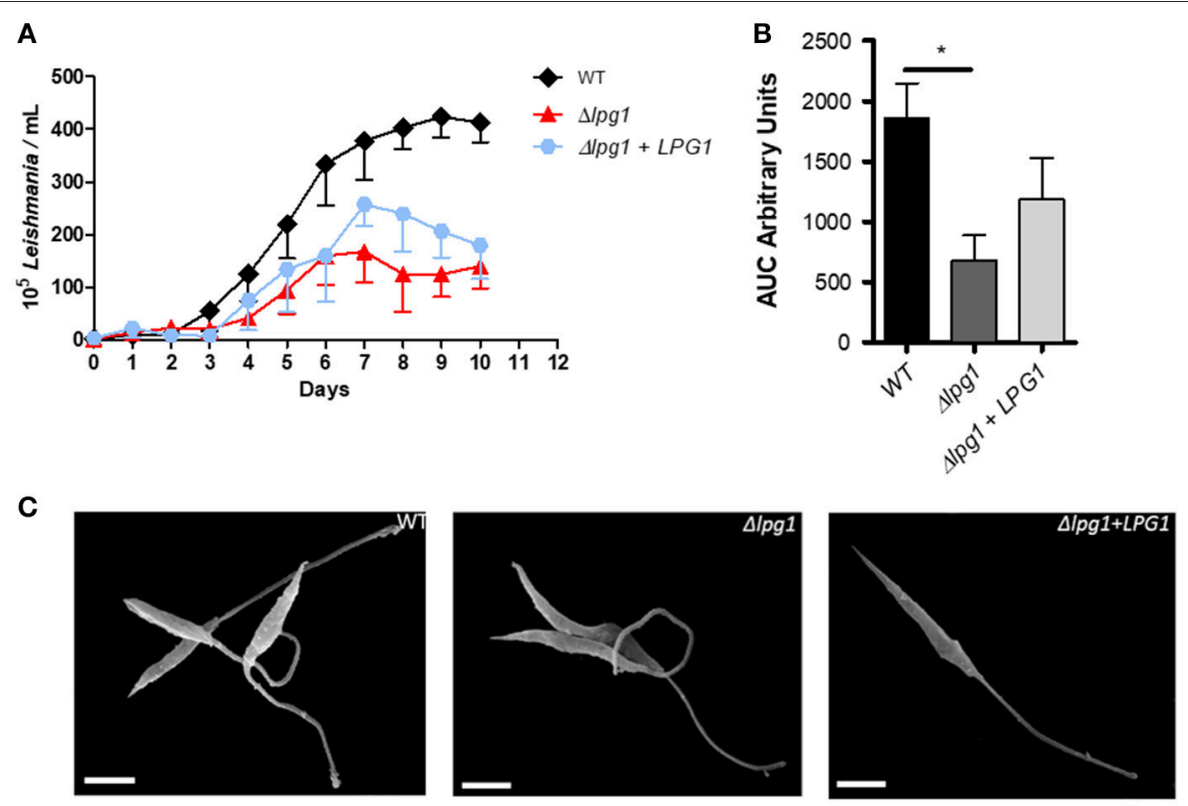

FIGURE 2 | Growth curve and morphology of the $\Delta / p g 1$ mutant. WT, $\Delta / p g 1$ and $\Delta / p g 1+L P G 1$ parasites were cultured at initial concentrations of $1 \times 10^{5} / \mathrm{ml}$ in HOMEM medium. (A) Axenic growth curve of late log-phase WT, $\Delta / p g 1$ and $\Delta / p g 1+L P G 1$ parasites, as showed by the area under the curve (AUC) (B). The number of viable parasites was evaluated by direct counting. Each point represents mean and SE. Data are representative of at least three independent assays and were collected in triplicate for each condition. ${ }^{*} p<0.05$. (C) Parasites were processed for scanning electron microscopy (SEM) and photographed under a JEOL JSM-6390LV microscope at 6000x magnification (C). Scale bar, $2 \mu \mathrm{m}$.
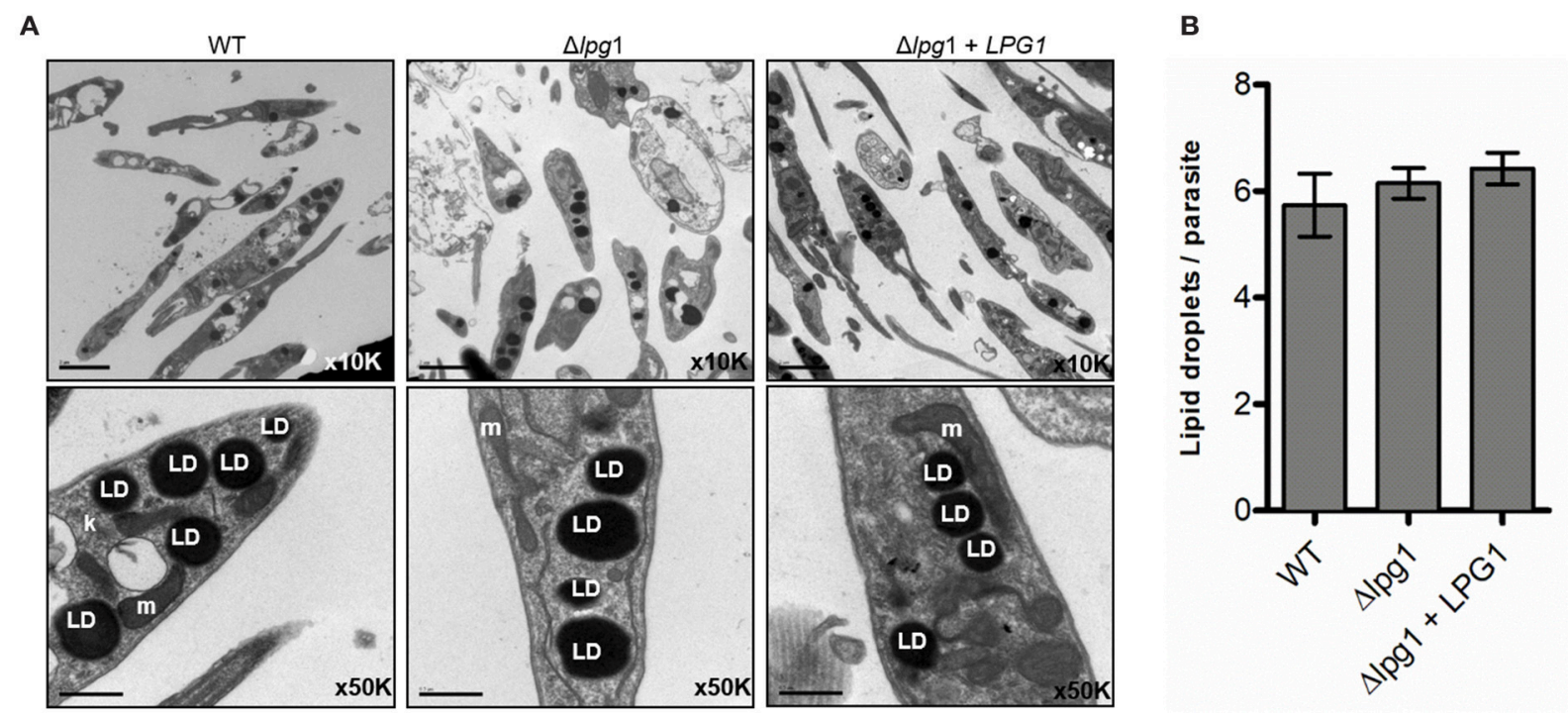

FIGURE 3 | Deletion of LPG1 does not alter LD formation in Leishmania infantum. (A) Panels show stationary phase WT, $\triangle / p g 1$ and $\triangle / p g 1+L P G 1$ promastigotes analyzed by transmission electron microscopy (TEM) and photographed under a JEOL 1230 microscope. (B) Bars represent the mean number of LD \pm SE in WT, $\Delta / p g 1$ or $\Delta / p g 1+L P G 1$ parasites stained with osmium tetroxide. $\mathrm{k}$, kinetoplast; LD, lipid droplets; $\mathrm{m}$, mitochondrion. Scale bar, $0.5 \mu \mathrm{m}$.

$72 \mathrm{~h}$. No differences were observed between the Leishmania parasites after $4 \mathrm{~h}$ of infection (Figure 4). However, at $72 \mathrm{~h}$ post-infection, WT parasites survived more efficiently than the $\Delta l p g 1$ mutant. Expression of $L P G 1$ in the $\Delta l p g 1$ mutant partially restored its capacity to survive and replicate within macrophages. These data reinforce the importance of LPG as a virulence factor in the successful maintenance of Leishmania infantum infection. 


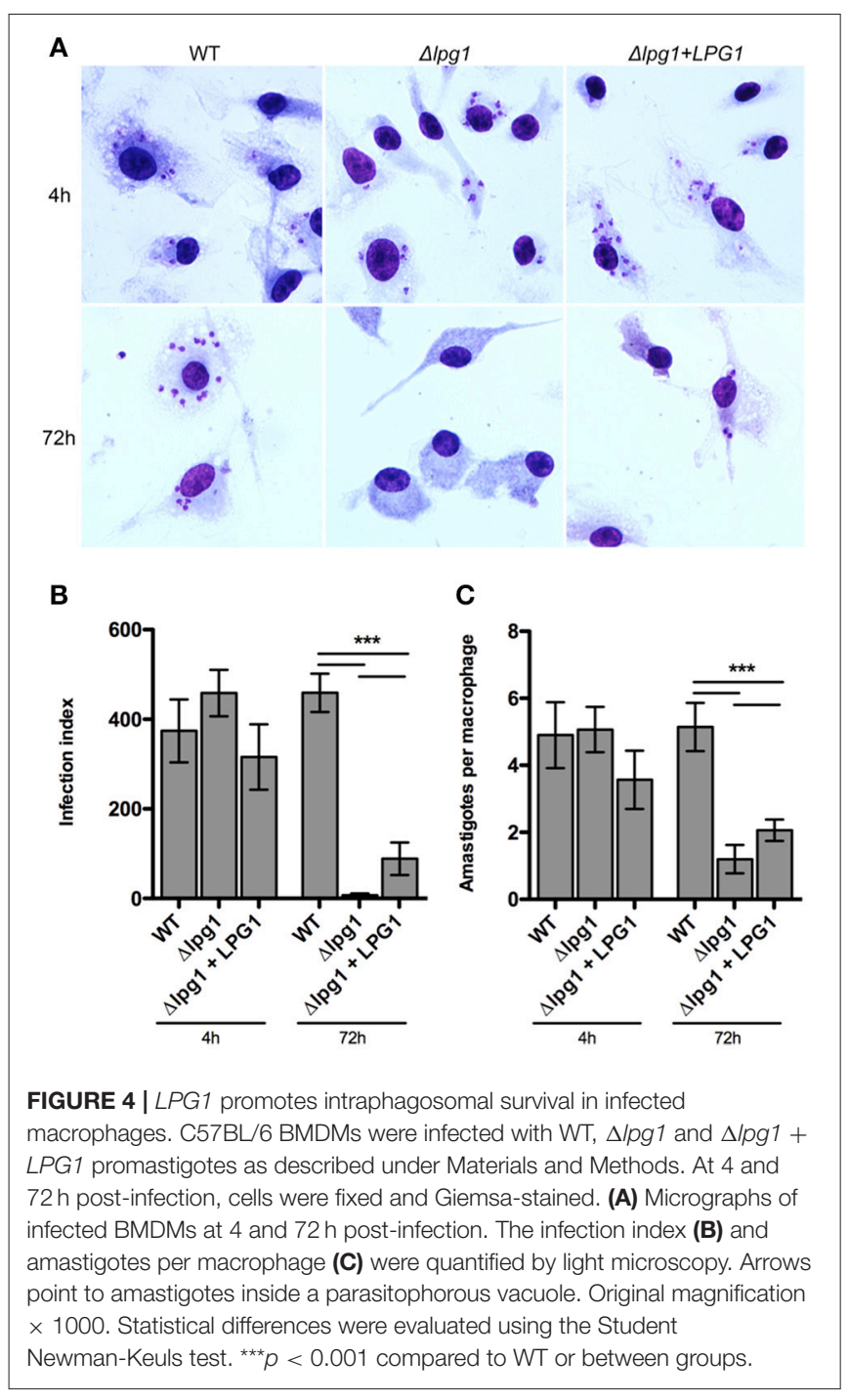

\section{LPG1-Null Mutants Induce NF- $\kappa$ B-Dependent iNOS Expression in Macrophages}

To assess the impact of LPG on the expression of inducible nitric oxide synthase by host cells, we first analyzed iNOS transcript levels in RAW 264.7 cells infected with WT or transgenic parasites. The $\Delta l p g 1$ mutant induced a robust (3.5fold increase) expression of iNOS compared to WT and $\Delta l p g 1+$ LPG1 promastigotes (Figure 5A). We next performed luciferase reporter assays to characterize the modulation of the iNOS promoter by L. infantum promastigotes. RAW 264.7 cells were transiently transfected with either the iNOS promoter reporter construct pTK-3XNS or the NF- $\kappa \mathrm{B}$ consensus luciferase reporter construct (p6кB-Luc) prior to infection with either L. infantum WT, $\Delta l p g 1$ or $\Delta l p g 1+L P G 1$, or stimulation with LPS. As shown in Figures 5B,C, LPG-deficient promastigotes induced stronger activation of the iNOS promoter and of the NF- $\kappa$ B reporter. Collectively, these findings indicate that LPG contributes to the evasion of iNOS expression by L. infantum promastigotes.

\section{DISCUSSION}

Previous studies using purified LPG were important to unravel its impact on the activation of the immune system. Although purified LPG from different species can activate the release of inflammatory mediators, understanding the role of this response in the context of infection remains a challenge. While some groups have characterized Old World LPG1defective Leishmania species, the behavior of these parasites when compared to their WT counterparts varies depending on the species under study (Spath et al., 2003; Capul et al., 2007; Forestier et al., 2014). Here, we generated for the first time a LPG-deficient mutant of $L$. infantum, a New World species cluster.

A comparison of parasite growth between axenic cultures containing each of the three isolates showed that the deletion of LPG1 resulted in a delayed capability of the $\Delta l p g 1$ mutant parasites to replicate in comparison to cultures of WT and $\Delta l p g 1$ + LPG1 parasites. While the deletion of the LPG1 gene had a limited impact on $L$. infantum promastigote proliferation, no significant morphological or ultrastructural alterations were seen in these parasites, indicating that targeting the LPG1 gene does not interfere with the intrinsic cell biology of L. infantum.

Recently, we have demonstrated that intact LPG from L. infantum promastigotes, but not its glycan and lipid moieties, induced a range of pro-inflammatory responses, including prostaglandin $\mathrm{E}_{2}\left(\mathrm{PGE}_{2}\right)$ and nitric oxide $(\mathrm{NO})$ release, increased LD formation, and inducible nitric oxide synthase (iNOS) and cyclooxygenase-2 expression (Lima et al., 2017). Consequently, a limitation of using purified LPG is that the physiological conditions present in host cell-parasite interactions are not accurately replicated. LDs are key cytoplasmic organelles involved in production of lipid mediators and pro-inflammatory cytokines in mammalian cells (Bozza et al., 2011). Several intracellular pathogens, including Leishmania, take advantage of LD formation in host cells (Rabhi et al., 2016). Moreover, LDs have also been described in trypanosomatids in association with arachidonic acid metabolism (Araújo-Santos et al., 2014). Our group previously reported an increase in LD formation during $L$. infantum metacyclogenesis, as well as in the intracellular amastigote form (Araújo-Santos et al., 2014). Here, we showed that the absence of the LPG1 gene in L. infantum did not alter the biogenesis of LDs. In addition, our previous findings showed that parasite-derived $\mathrm{PGF}_{2 \alpha}$ produced inside LDs plays a critical role during macrophage infection (Araújo-Santos et al., 2014). We fully intend to comprehensively investigate the potential influence of $L P G 1$ on the release of $\mathrm{PGF}_{2 \alpha}$ in infected macrophages using this novel L. infantum $\Delta l p g 1$ mutant.

In $L$. major and $L$. donovani, the specific loss of LPG through the ablation of LPG1 galactofuranosyl transferase strongly impairs the ability of parasites to survive within the sandfly host, as well as to establish infection in mammalian macrophages and in mice (Sacks et al., 2000; Spath et al., 2003; Secundino et al., 2010). Hence, in these species, LPG impairs the microbicidal mechanisms associated with the biogenesis of phagolysosomes, including assembly of the NADPH oxidase and recruitment of the v-ATPase (Lodge et al., 

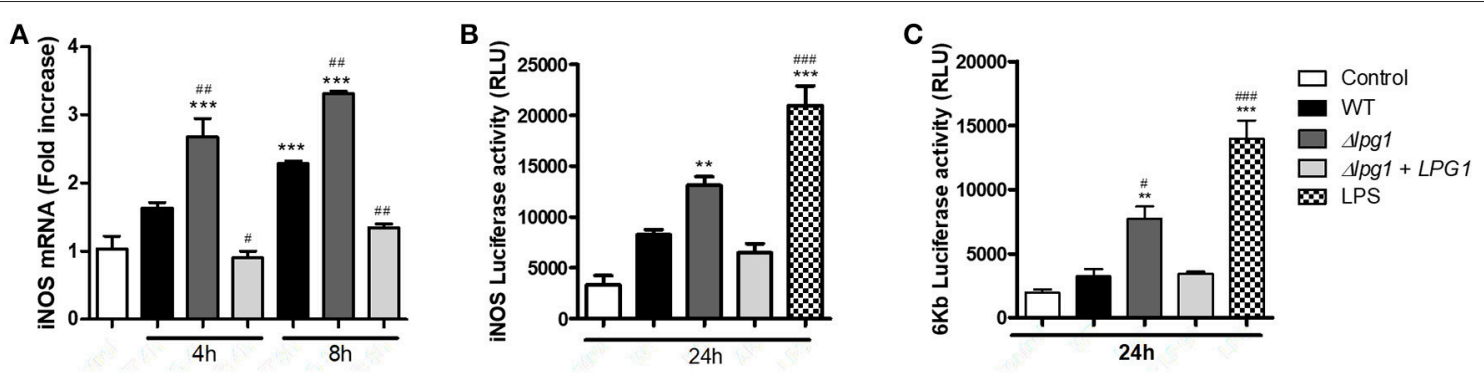

FIGURE 5 | L. infantum promastigotes evade NF-кB-dependent iNOS in an LPG-dependent manner in RAW 264.7 cells. RAW 264.7 cells were infected with either WT, $\Delta / p g 1$ or $\Delta / p g 1+L P G 1$ promastigotes. After 4 or $8 \mathrm{~h}$ of infection, iNOS expression was determined by qPCR (A). RAW 264.7 cells were transfected with either

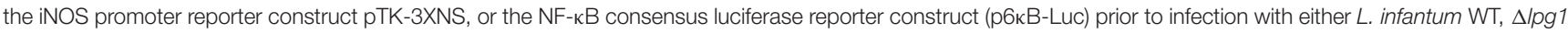
or $\Delta / p g 1+L P G 1$, or stimulation with LPS. At $24 \mathrm{~h}$ post-infection, activity of the iNOS promoter (B) and the NF-kB reporter (C) was measured by quantification of luciferase activity (B). Bars represent means \pm SE of three representative experiments performed in triplicate for murine cells. ${ }^{\star \star \star} p<0.0001$; ${ }^{\star \star} p<0.001$ compared to control. \#\#\# $p<0.0001$, \#\# $p<0.001$ and $\# p<0.05$ compared to WT. $P<0.0001$ compared with control group Student Newman-Keuls post-test.

2006; Vinet et al., 2009). Our results indicate that, similarly to these species, L. infantum LPG1 is required for replication within macrophages. Whether LPG contributes to the ability of L. infantum to successfully infect macrophages through the impairment of phagolysosomal biogenesis remains to be investigated. Interestingly, we observed that our $\Delta l p g 1$ mutant parasites induced robust NF- $\kappa \mathrm{B}$-dependent iNOS expression compared to parental WT L. infantum promastigotes. This seems to suggest that the reduced survival of $\Delta l p g 1$ mutants in mouse macrophages may be related to higher levels of iNOS, which is responsible for the generation of leishmanicidal nitric oxide (Coelho-Finamore et al., 2011; Passero et al., 2015). Further study will involve investigating the contribution of nitric oxide production with respect to the reduced ability of $\Delta l p g 1$ mutants to survive within macrophages. The underlying mechanism by which $\Delta$ lpg1 mutant parasites induce high levels of NF- $\kappa$ B activation remains unknown, and thus represents an additional aspect of host cell-parasite interplay that we intend to further investigate.

With regard to the partial restoration of the WT phenotype observed in $\triangle$ lpg1 + LPG1 parasites, it has been welldocumented that complemented parasites commonly do not fully recover virulence. The inappropriate regulation of LPG1 expression by the episomal vector may be a possible explanation for this observation (Späth et al., 2000; Spath et al., 2003; Joshi et al., 2002). A previous study demonstrated that L. major LPG1deficient mutant promastigotes present an attenuated virulence phenotype, as evidenced by the delayed formation of lesions in vivo (Späth et al., 2000). In addition, this delay was associated with a 100-fold decrease in parasite survival within macrophages in vitro. The data presented herein are consistent with these results, as well as with other reports in the literature (Privé and Descoteaux, 2000; Sacks et al., 2000; Zhang et al., 2004) propounding LPG as a virulence factor.

Taken together, the present findings support the importance of creating LPG-deficient mutants in various Leishmania spp. as a unique tool to investigate the specific impact and contribution of this abundant virulence factor in the complex host cellLeishmania interplay. Hence, we are currently conducting studies to compare the responses of various immune cells to live L. infantum promastigotes in the presence or absence of surfaceexpressed LPG, since we feel it is important to thoroughly characterize these isolates to obtain a more comprehensive understanding regarding the role of L. infantum LPG1 in future in vitro and in vivo studies.

\section{AUTHOR CONTRIBUTIONS}

ML-S, CM, JL, GA, GQ-C, AC, SM-P, UG, LF, TA-S, AD, and $\mathrm{VB}$ conceived and designed the study, contributed to the data analysis, drafted, and revised the manuscript. ML-S, CM, JL, GA, GQ-C, AC, SM-P, CF, FJ-S, and TA-S performed the experiments. ML-S, CM, JL, LF, TA-S, AD, and VB wrote and revised the manuscript. All authors read and approved the final version of this manuscript.

\section{FUNDING}

This work was supported by the Brazilian National Research Council (CNPq), (Grant number: 400135/2014-0 to VB), Fundação de Amparo à Pesquisa do Estado da Bahia (FAPESB) (Grant number: 04/2015 to VB), Science without Borders (SwB) (to $\mathrm{VB}$ and $\mathrm{AD}$ ) and the Canadian Institutes of Health Research (CIHR) (grant MOP-125990 to AD). VB and UG are senior investigator funded by $\mathrm{CNPq}$. $\mathrm{AD}$ is the holder of the Canada Research Chair on the Biology of intracellular parasitism. GA was partially supported by a CIHR Frederick Banting and Charles Best Doctoral Award. AC: received a fellowship from CNPq. The funders had no role in study design, data collection or analysis, the decision to publish, or preparation of the manuscript.

\section{ACKNOWLEDGMENTS}

The authors thank Andrezza Souza for technical and logistics support. The authors would like to thank Andris K. Walter for English language revision and manuscript copyediting assistance. 


\section{REFERENCES}

Araújo-Santos, T., Rodríguez, N. E., Moura-Pontes, S., Dixt, U. G., Abánades, D. R., Bozza, P. T., et al. (2014). Role of prostaglandin F2 $\alpha$ production in lipid bodies from Leishmania infantum chagasi: insights on virulence. J. Infect. Dis. 210, 1951-1961. doi: 10.1093/infdis/jiu299

Becker, I., Salaiza, N., Aguirre, M., Delgado, J., Carrillo-Carrasco, N., Kobeh, L. G., et al. (2003). Leishmania lipophosphoglycan (LPG) activates NK cells through toll-like receptor-2. Mol. Biochem. Parasitol. 130, 65-74. doi: 10.1016/S0166-6851(03)00160-9

Bozza, P. T., Bakker-Abreu, I., Navarro-Xavier, R. A., and Bandeira-Melo, C. (2011). Lipid body function in eicosanoid synthesis: an update. Prostaglandins Leukot Essent Fat Acids 85, 205-213. doi: 10.1016/j.plefa.2011. 04.020

Capul, A. A., Hickerson, S., Barron, T., Turco, S. J., and Beverley, S. M. (2007). Comparisons of mutants lacking the golgi UDP-galactose or GDP-mannose transporters establish that phosphoglycans are important for promastigote but not amastigote virulence in Leishmania major. Infect. Immun. 75, 4629-4637. doi: 10.1128/IAI.00735-07

Coelho-Finamore, J. M., Freitas, V. C., Assis, R. R., Melo, M. N., Novozhilova, N., Secundino, N. F., et al. (2011). Leishmania infantum: Lipophosphoglycan intraspecific variation and interaction with vertebrate and invertebrate hosts. Int. J. Parasitol. 41, 333-342. doi: 10.1016/j.ijpara.2010.10.004

Dermine, J. F., Scianimanico, S., Priv,é, C., Descoteaux, A., and Desjardins, M. (2000). Leishmania promastigotes require lipophosphoglycan to actively modulate the fusion properties of phagosomes at an early step of phagocytosis. Cell. Microbiol. 2, 115-126. doi: 10.1046/j.1462-5822.2000.00037.x

Descoteaux, A., Avila, H. A., Zhang, K., Turco, S. J., and Beverley, S. M. (2002). Leishmania LPG3 encodes a GRP94 homolog required for phosphoglycan synthesis implicated in parasite virulence but not viability. EMBO J. 21, 4458-4469. doi: 10.1093/emboj/cdf447

Descoteaux, A., Luo, Y., Turco, S. J., and Beverley, S. M. (1995). A specialized pathway affecting virulence glycoconjugates of Leishmania. Science 269, 1869-1872. doi: 10.1126/science.7569927

Descoteaux, A., Mengeling, B. J., Beverley, S. M., and Turco, S. J. (1998). Leishmania donovani has distinct mannosylphosphoryltransferases for the initiation and elongation phases of lipophosphoglycan repeating unit biosynthesis. Mol. Biochem. Parasitol. 94, 27-40. doi: 10.1016/S0166-6851(98)00047-4

Descoteaux, A., and Turco, S. J. (1993). The lipophosphoglycan of Leishmania and macrophage protein kinase C. Parasitol. Today 9, 468-471. doi: 10.1016/0169-4758(93)90105-O

Descoteaux, A., Turco, S. J., Sacks, D. L., and Matlashewski, G. (1991). Leishmania donovani lipophosphoglycan selectively inhibits signal transduction in macrophages. J Immunol 146, 2747-2753.

Desjardins, M., and Descoteaux, A. (1997). Inhibition of phagolysosomal biogenesis by the Leishmania lipophosphoglycan. J. Exp. Med. 185, 2061-2068. doi: 10.1084/jem.185.12.2061

de Veer, M. J., Curtis, J. M., Baldwin, T. M., DiDonato, J. A., Sexton, A., McConville, M. J., et al. (2003). MyD88 is essential for clearance of Leishmania major: possible role for lipophosphoglycan and Toll-like receptor 2 signaling. Eur. J. Immunol. 33, 2822-2831. doi: 10.1002/eji.200324128

Forestier, C. L., Gao, Q., and Boons, G. J. (2014). Leishmania lipophosphoglycan: how to establish structure-activity relationships for this highly complex and multifunctional glycoconjugate? Front. Cell. Infect. Microbiol. 4:193. doi: $10.3389 /$ fcimb. 2014.00193

Ilg, T. (2000). Lipophosphoglycan is not required for infection of macrophages or mice by Leishmania mexicana. EMBO J. 19, 1953-1962. doi: 10.1093/emboj/19.9.1953

Ilg, T., Demar, M., and Harbecke, D. (2001). Phosphoglycan Repeat-deficient Leishmania mexicana parasites remain infectious to macrophages and mice. J. Biol. Chem. 276, 4988-4997. doi: 10.1074/jbc.M008030200

Ilg, T., Montgomery, J., Stierhof, Y. D., and Handman, E. (1999). Molecular cloning and characterization of a novel repeat-containing Leishmania major gene, ppgl, that encodes a membrane-associated form of proteophosphoglycan with a putative glycosylphosphatidylinositol anchor. J. Biol. Chem. 274, 31410-31420. doi: $10.1074 /$ jbc. 274.44 .31410
Joshi, P. B., Kelly, B. L., Kamhawi, S., Sacks, D. L., and McMaster, W. R. (2002). Targeted gene deletion in Leishmania major identifies leishmanolysin (GP63) as a virulence factor. Mol. Biochem. Parasitol. 120, 33-40. doi: 10.1016/S0166-6851(01)00432-7

Kavoosi, G., Ardestani, S. K., and Kariminia, A. (2009). The involvement of TLR2 in cytokine and reactive oxygen species (ROS) production by PBMCs in response to Leishmania major phosphoglycans (PGs). Parasitology 136, 1193-1199. doi: 10.1017/S0031182009990473

Lima, J. B., Araújo-Santos, T., Lázaro-Souza, M., Carneiro, A. B., Ibraim, I. C., Jesus-Santos, F. H., et al. (2017). Leishmania infantum lipophosphoglycan induced-Prostaglandin E2 production in association with PPAR- $\gamma$ expression via activation of Toll like receptors-1 and 2. Sci. Rep. 7:14321. doi: 10.1038/s41598-017-14229-8

Lodge, R., Diallo, T. O., and Descoteaux, A. (2006). Leishmania donovani lipophosphoglycan blocks NADPH oxidase assembly at the phagosome membrane. Cell. Microbiol. 8, 1922-1931. doi: 10.1111/j.1462-5822.2006.00758.x

McConville, M. J., and Ferguson, M. A. (1993). The structure, biosynthesis and function of glycosylated phosphatidylinositols in the parasitic protozoa and higher eukaryotes. Biochem. J. 294, 305-324. doi: 10.1042/bj2940305

Moradin, N., and Descoteaux, A. (2012). Leishmania promastigotes: building a safe niche within macrophages. Front. Cell. Infect. Microbiol. 2:121. doi: 10.3389/fcimb.2012.00121

Passero, L. F., Assis, R. R., da Silva, T. N., Nogueira, P. M., Macedo, D. H., Pessoa, N. L., et al. (2015). Differential modulation of macrophage response elicited by glycoinositolphospholipids and lipophosphoglycan from Leishmania (Viannia) shawi. Parasitol. Int. 64, 32-35. doi: 10.1016/j.parint.2015.01.006

Privé, C., and Descoteaux, A. (2000). Leishmania donovani promastigotes evade the activation of mitogen-activated protein kinases p38, c-Jun N-terminal kinase, and extracellular signal-regulated kinase-1/2 during infection of naive macrophages. Eur. J. Immunol. 30, 2235-2244. doi: 10.1002/15214141(2000)30:8\&lt;2235::AID-IMMU2235\&gt;3.0.CO;2-9

Rabhi, S., Rabhi, I., Trentin, B., Piquemal, D., Regnault, B., Goyard, S., et al. (2016). Lipid droplet formation, their localization and dynamics during leishmania major macrophage infection. PLoS ONE 11:e0148640. doi: 10.1371/journal.pone.0148640

Rojas-Bernabé, A., Garcia-Hernández, O., Maldonado-Bernal, C., DelegadoDominguez, J., Ortega, E., Gutiérrez-Kobeh, L., et al. (2014). Leishmania mexicana lipophosphoglycan activates ERK and p38 MAP kinase and induces production of proinflammatory cytokines in human macrophages through TLR2 and TLR4. Parasitology 141, 788-800. doi: 10.1017/S00311820130 02187

Ryan, K. A., Garraway, L. A., Descoteaux, A., Turco, S. J., and Beverley, S. M. (1993). Isolation of virulence genes directing surface glycosylphosphatidylinositol synthesis by functional complementation of Leishmania. Proc. Natl. Acad. Sci. U.S.A. 90, 8609-8613. doi: 10.1073/pnas.90. 18.8609

Sacks, D., and Kamhawi, S. (2001). Molecular aspects of parasite-vector and vector-host interactions in Leishmaniasis. Annu. Rev. Microbiol. 55, 453-483. doi: 10.1146/annurev.micro.55.1.453

Sacks, D. L., Modi, G., Rowton, E., Späth, G., Epstein, L., Turco, S. J., et al. (2000). The role of phosphoglycans in Leishmania-sand fly interactions. Proc. Natl. Acad. Sci. U.S.A. 97, 406-411. doi: 10.1073/pnas.97.1.406

Secundino, N., Kimblin, N., Peters, N. C., Lawyer, P., Capul, A. A., Beverley, S. M., et al. (2010). Proteophosphoglycan confers resistance of Leishmania major to midgut digestive enzymes induced by blood feeding in vector sand flies. Cell. Microbiol. 12, 906-918. doi: 10.1111/j.1462-5822.2010.01439.x

Späth, G. F., Epstein, L., Leader, B., Singer, S. M., Avila, H. A., Turco, S. J., et al. (2000). Lipophosphoglycan is a virulence factor distinct from related glycoconjugates in the protozoan parasite Leishmania major. Proc. Natl. Acad. Sci. U.S.A. 97, 9258-9263. doi: 10.1073/pnas.160257897

Spath, G. F., Garraway, L. A., Turco, S. J., and Beverley, S. M. (2003). The role(s) of lipophosphoglycan (LPG) in the establishment of Leishmania major infections in mammalian hosts. Proc. Natl. Acad. Sci.U.S.A. 100, 9536-9541. doi: 10.1073/pnas. 1530604100

Tavares, N. M., Araújo-Santos, T., Afonso, L., Nogueira, P. M., Lopes, U. G., Soares, R. P., et al. (2014). Understanding the mechanisms controlling 
Leishmania amazonensis infection in vitro: the role of LTB4 derived from human neutrophils. J. Infect. Dis. 210, 656-666. doi: 10.1093/infdis/jiu158

Turco, S., Descoteaux, A., Ryan, K., Garraway, L., and Beverley, S. (1994). Isolation of virulence genes directing GPI synthesis by functional complementation of Leishmania. Brazilian J. Med. Biol. Res. 27, 133-138.

Turco, S. J., and Descoteaux, A. (1992). The lipophosphoglycan of leishmania parasites. Annu. Rev. Microbiol. 46, 65-94. doi: 10.1146/annurev.mi.46.100192.000433

Vinet, A. F., Fukuda, M., Turco, S. J., and Descoteaux, A. (2009). The Leishmania donovani lipophosphoglycan excludes the vesicular proton-ATPase from phagosomes by impairing the recruitment of Synaptotagmin, V. PLoS Pathog. 5:e1000628. doi: 10.1371/journal.ppat.1000628

Zhang, K., Barron, T., Turco, S. J., and Beverley, S. M. (2004). The LPG1 gene family of Leishmania major. Mol. Biochem. Parasitol. 136, 11-23. doi: 10.1016/j.molbiopara.2004.02.012
Conflict of Interest Statement: The authors declare that the research was conducted in the absence of any commercial or financial relationships that could be construed as a potential conflict of interest.

The reviewer LM declared a shared affiliation, with no collaboration, with two of the authors, AC and UG, to the handling Editor.

Copyright $\odot 2018$ Lázaro-Souza, Matte, Lima, Arango Duque, Quintela-Carvalho, de Carvalho Vivarini, Moura-Pontes, Figueira, Jesus-Santos, Gazos Lopes, Farias, Araújo-Santos, Descoteaux and Borges. This is an open-access article distributed under the terms of the Creative Commons Attribution License (CC BY). The use, distribution or reproduction in other forums is permitted, provided the original author(s) and the copyright owner are credited and that the original publication in this journal is cited, in accordance with accepted academic practice. No use, distribution or reproduction is permitted which does not comply with these terms. 